\title{
Diagnostics as the first line of defense in global health security
}

\author{
Mark Kessel
}

\begin{abstract}
The creation of the Global Health Security Agenda is a key step in the goal of combatting outbreaks in an increasingly interconnected world. It is too bad that the initiative is so woefully underfunded.
\end{abstract}

\begin{abstract}
Countless dollars have been deployed across the globe in the 'war on terror'. In the United States alone, tens of billions of dollars have been spent on biodefense funding programs to create countermeasures to bioterrorism threats, many of which may never arise. In the meantime, many public health authorities have lost their focus on a clear and ever present danger-the spread of old scourges and emerging pathogens in an increasingly interconnected world. Infectious diseases do not respect national borders. People traveling around the world now pose greater potential biological threats than they did in previous generations. From a world health perspective, much as in the war on terror, the most effective way to deal with such threats is at their source. To do so effectively, diagnostics are needed that can rapidly and accurately identify the infections and promote surveillance to halt their further spread from country to country. With this in mind, the recent creation of the Global Health Security Agenda (GHSA) by 27 countries represents an important step in tracking the spread of infection and enabling the deployment of effective countermeasures. It is too bad that it is being so pitifully funded that it is likely to prove ineffective in reaching its important goals.
\end{abstract}

\section{Clear and present danger}

It is evident that the world health community is losing the battle against infectious diseases.

Mark Kessel is chairman of the Foundation for Innovative New Diagnostics (FIND), of counsel to Shearman and Sterling LLP and a founding partner of Symphony Capital LLP, New York, NY, USA.

e-mail:mark.kessel@shearman.com

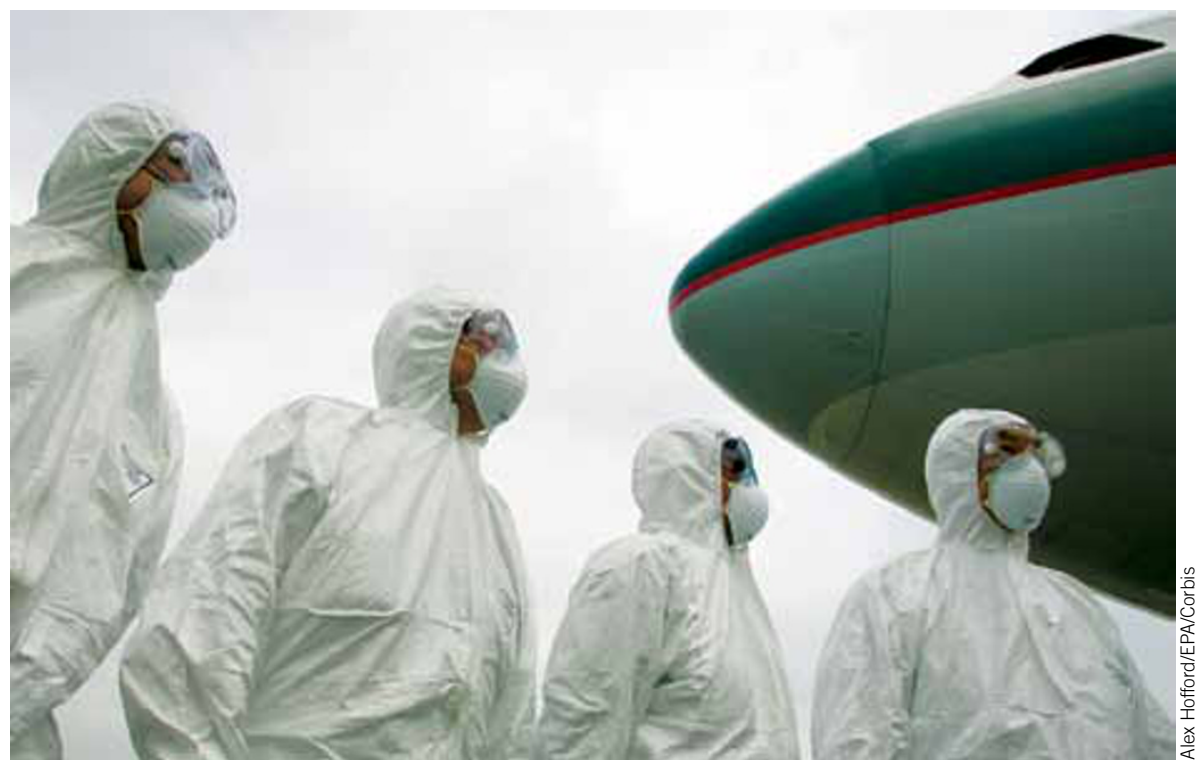

As the globalized world becomes increasingly interconnected, the specter of infectious disease will require much more investment in diagnostics to track and identify pathogenic agents.

Bacteria are becoming resistant to modern medicine in developed and developing countries. In addition, political and religious factors, population growth and climate change are also affecting global health security. In Pakistan, the Taliban is impeding the eradication of polio in its areas of influence by resorting to violence and misinformation to prevent the vaccination of children. Upheaval and population movement in conflict-rife areas of central Africa are creating living conditions in refugee camps that encourage the spread of infections.

To illustrate the havoc to global health security that merely one individual can create, consider the case in 2007 of the Atlanta-based personal-injury lawyer Andrew Speaker who, while infected with a strain of tuberculosis, boarded a flight in the United States and then traveled to Paris, Greece, Italy, Prague, Montreal and finally back to the United States. To contain the health threat he posed, the US Centers for Disease Control, believing he was infected with extensively drug-resistant tuberculosis (XDR-TB), placed him under involuntary isolation by invoking the US Public Health Service Act. XDR-TB is complicated to treat and can be lethal-a 2006 outbreak in a village in South Africa resulted in 52 out of 53 infected individuals dying within months. The Ebola outbreak in Guinea in March of this year quickly claimed 84 lives as the outbreak jumped national 
borders to Sierra Leone, Liberia and Mali and prompted a public health scare in Canada when a citizen who had traveled in Liberia showed symptoms of disease. The World Health Organization (WHO) believes that the spread of the disease to the capital of Guinea was caused by a single person who had been infected in a remote area of the country, more than 200 miles away. Recently, Middle East respiratory syndrome, a dangerous new virus, was detected in Florida after having traveled to the United Kingdom, France and Malaysia.

Aside from their impact on public health, disease threats can be devastating to the global economy. It is estimated that the outbreak of severe acute respiratory syndrome (SARS) in 2002-2003 alone cost the world economy $\$ 40$ billion in just four months. Resourceconstrained countries that have high incidence rates of several infectious diseases, such as AIDS and TB, suffer an especially severe public health and economic burden.

\section{Taking action}

Steps have been taken to deal with global health security but have not come close to resolving the enormity of the threat. For example, all WHO members have signed up to the International Health Regulations. Even so, fewer than one-fifth have achieved full compliance with these regulations. Thus, the vast majority are not fully prepared to detect and respond to infectious disease threats.

In February, as recognition of these shortcomings, the United States and 26 other countries commenced the GHSA. This initiative has the central goal of preventing and addressing infectious disease outbreaks before they spread around the globe. The focus is on improving disease monitoring and developing tests for various pathogens, and diagnostics are recognized as a major ingredient to this effort.

One objective of the agenda is to strengthen national and regional capacity at points of care and points of need to enable accurate, timely collection and analysis of information and to help develop laboratory systems capable of safely and accurately detecting all major pathogens with minimal risk.

\section{Numbers that do not add up}

Toward this lofty goal, the GHSA has allocated a mere $\$ 40$ million for 2014 , and next year, it is seeking little more ( $\$ 45$ million). This is not nearly enough to accomplish the agenda's goals, or even the objective enunciated for just the diagnostic component.

Consider the threat that $\mathrm{TB}$ alone poses to world health. More than 20 countries have been identified as having a high incidence of TB. It is estimated that 8.6 million people contract TB each year and about 1.5 million die of it each year. Because it spreads between people who come in contact with each other, its control depends on the rapid identification and treatment of active cases. As the Speaker case illustrates, the ability to discern whether an individual infected with $\mathrm{TB}$ carries a multidrug-resistant strain or XDR-TB is crucial not only for proper treatment but also for the protection of those with whom he or she has come in contact and to prevent the further

Public and philanthropic
funding for diagnostics for the
developing world totaled a mere
$\$ 118$ million in 2011 , well below
the level needed to address the
threat to global health security
posed by these diseases.

spread of the infection around the globe. Therefore, even if new, effective drugs were to be developed, there is still a need for improved detection with diagnostics to shorten the time to diagnosis with increased accuracy.

There are many challenges to developing and successfully introducing a TB diagnostic in these countries. Apart from the need to be inexpensive, a diagnostic must perform to high standards in difficult environments, such as villages across much of Africa and other parts of the world where only a small percentage of health facilities have access to a reliable power source. For those settings, creating incentives for manufacturers to develop a diagnostic poses a substantial challenge. The test must meet the characteristics required by the $\mathrm{WHO}$ and be 'affordable, sensitive, specific, userfriendly, robust and rapid, equipment-free and deliverable' (ASSURED). At the same time, the successful introduction in those countries requires the collaboration of the healthcare industry and local governmental health ministries. Thus, for the diseases most likely to spread infection around the world, manufacturers are not clamoring to develop diagnostics for these markets.

\section{Diagnostics - the 'ugly stepchild' of global health}

The GHSA makes a clear case that there is a need for a partnership with nations, international organizations and public and private stakeholders. Few of these have ever given diagnostics the kind of funding needed to address one of these threats, no less the entire threat of infectious diseases. In the interconnected world of the twentyfirst century, this threat has never been more serious.

Public and philanthropic funding for diagnostics for the developing world totaled a mere $\$ 118$ million in 2011, well below the level needed to address the threat to global health security posed by these diseases. Although less costly than therapeutics, an effective complex diagnostic can still cost $\$ 50$ million to develop. If governments are truly concerned about global health security, they will need to greatly increase funding for reliable diagnostics, especially for those that will be suitable for use in low-resource countries where many of the threats to global health are likely to originate.

In the absence of a concerted effort to fund diagnostics, the terror infectious diseases can inflict upon the world will not be contained. And the vision of the GHSA to provide us a world safe and secure from global health threats will never become a reality.

\section{COMPETING FINANCIAL INTERESTS}

The author declares no competing financial interests. 